\title{
Dynamic CT Perfusion Imaging: State of the Art
}

pISSN 2508-707X / elSSN 2508-7088 https://doi.org/10.22468/cvia.2018.00031 CVIA 2018;2(2):38-48

Received: March 3, 2018

Revised: April 3, 2018

Accepted: April 10, 2018

Corresponding author

Kakuya Kitagawa, MD, PhD

Department of Radiology, Mie University

School of Medicine, 2-174 Edobashi, Tsu,

Mie 514-8507, Japan

Tel: 81-59-231-5029

Fax: 81-59-232-8066

E-mail: kakuya@clin.medic.mie-u.ac.jp

\author{
Kakuya Kitagawa, Yoshitaka Goto, Satoshi Nakamura, \\ Masafumi Takafuji, Ahmed Hamdy, \\ Masaki Ishida, Hajime Sakuma \\ Department of Radiology, Mie University School of Medicine, Tsu, Japan
}

Recent advances in computed tomography (CT) technology have enabled stress dynamic CT perfusion (CTP) imaging of the entire myocardium to be performed with high temporal resolution and reasonable radiation dose. The most important advantage of dynamic CTP is the feasibility of complete quantification of myocardial perfusion in $\mathrm{mL} / \mathrm{min} / \mathrm{g}$. Although high diagnostic values have been reported for the detection of myocardial ischemia, standardization of acquisition method, mathematical modeling and interpretation, as well as further evaluation of its accuracy, clinical role, and cost effectiveness are required before its routine clinical implementation. The current review focuses on the state-of-the-art of dynamic CTP and outlines its current status and future directions as a new technology for the physiologic assessment of coronary artery disease.

Key words Multidetector computed tomography - Myocardial perfusion imaging · Coronary artery disease.

\section{INTRODUCTION}

Over the last two decades, computed tomography (CT) has made significant advancement. Coronary CT angiography (CCTA), which first appeared in 2001 in the literature, is now widely performed for patients with suspected and known coronary artery disease (CAD). CCTA has high sensitivity (94-99\%) and specificity (64-83\%) and a negative predictive value (NPV) of $97-99 \%$ for detecting obstructive CAD [1]. A recent prospective multicenter randomized controlled trial that assessed the role of CCTA in $>4000$ patients with suspected angina due to coronary heart disease demonstrated that, when included in the initial assessment of chest symptoms, CCTA can contribute to more accurate diagnosis of $\mathrm{CAD}$, more efficient downstream testing, and more appropriate treatments [2]. More importantly, fatal and nonfatal myocardial infarction was halved in patients allocated to CCTA compared with those assigned to standard care [3]. In response to the results of the randomized trial, the National Institute for Health and Care Excellence in the United Kingdom updated its clinical guideline for chest pain of recent onset so that CCTA is recommended as the first-line test [4].

Although CCTA is a robust technique for assessing morpho-

(c) This is an Open Access article distributed under the terms of the Creative Commons Attribution Non-Commercial License (http://creativecommons.org/licenses/by$\mathrm{nc} / 4.0$ ) which permits unrestricted non-commercial use, distribution, and reproduction in any medium, provided the original work is properly cited. logical stenosis and plaque burden of coronary artery, it is limited in determining the hemodynamic significance of coronary stenoses. Solely with morphological assessment of stenosis, CCTA can provide only poor correlation with evidence of myocardial ischemia and a positive predictive value (PPV) of 29$58 \%$ [5]. Considering the growing evidence of the benefit of the physiology-guided coronary revascularization strategy [6-8], routine integration of anatomical information by CCTA and location, extent, and severity of ischemia should be valuable. Such information can be obtained through non-invasive functional imaging such as single photon emission computed tomography (SPECT), positron emission tomography (PET), or stress perfusion magnetic resonance imaging (MRI). However, $\mathrm{CT}$ is evolving to a standalone modality for comprehensive assessment of anatomical stenosis and its hemodynamic significance in the same examination due to the remarkable advance of CT systems and post-processing. Actually, CT-derived fractional flow reserve (FFR) and stress CT perfusion (CTP) imaging are currently under intensive investigation. Given different underlying physical principles and the types of pathophysiological information they provide, these techniques have their own advantages and disadvantages [9] and may have complementary clinical roles. But it should be noted that CTP is not a computer simulation, but assesses what is happening in vivo with unprecedented spatial resolution, which may contribute to advance 
our understanding of myocardial perfusion at the microcirculatory level.

There are currently two approaches in CTP-static and dynamic. Static CTP imaging refers to the assessment of myocardial enhancement acquired at a single time point of the first-pass of contrast material bolus. Since the first human report of CTP under adenosine stress by Kurata et al. [10], static CTP has been evaluated by many single and multicenter studies because of its technical ease of implementation-static CTP can be performed on any CT platform that is capable of CCTA. However, success of perfusion assessment by static CTP is highly dependent on contrast material bolus timing [11-14] and motion [15,16]. In contrast, with a dynamic approach, myocardial perfusion is assessed based on myocardial enhancement at multiple time points of first-pass of the contrast material, which is robust in terms of bolus timing and allows for fully quantitative analysis of myocardial perfusion. Although clinical implementation of dynamic CT perfusion has been a challenge because of its limited z-axis coverage $(\sim 4 \mathrm{~cm})$ of 64 -detector row $\mathrm{CT}$ scanners and relatively high radiation dose inherent to repetitive image acquisition, recent advances in CT technology allow dynamic CTP of the entire myocardium to be performed at reasonably reduced radiation exposure. The purpose of this review is to describe the state-of-the art of dynamic CTP including advantages and disadvantages of different CT platforms.

\section{PREREQUISITES FOR DYNAMIC CTP}

In order to obtain high quality dynamic CTP images, a high temporal resolution and wide detector coverage along the $\mathrm{z}$-axis are essential. In Table 1 , characteristics of commercially available CT scanners capable of dynamic CTP with wide $\mathrm{z}$-axis coverage are summarized.

\section{High temporal resolution}

The most common and basic approach to optimize temporal resolution for CCTA is half-scan reconstruction (180-degree reconstruction). However, reconstruction using a wider array of projections is advantageous for CT number accuracy since adding projections beyond $180^{\circ}$ improves the signal-to-noise ratio [17], reduces streak and partial scan artifacts [18-20], and enables reconstruction of wider $z$-axis coverage [21]. For dualsource CT scanners, $180^{\circ}\left(=90^{\circ}\right.$ rotation) and $360^{\circ}\left(=270^{\circ}\right.$ rotation) reconstruction are blended after filtering, thus preserving high temporal resolution of half scan and CT number stability of full scan $[19,22]$. However, $360^{\circ}$ rotation data are more commonly used for dynamic CTP with wide-detector CT scanners $[21,23,24]$. Considering that successful vasodilatorstress results in heart rate increase $>10$ beats per minute, current temporal resolution of multidetector CT ( $\geq 250 \mathrm{~ms}$ with fullscan reconstruction) requires further improvement [25]. Motion artifacts due to increased heart rate during adenosine stress have been observed in a significant percentage of patients in static CTP [16]. At present, systolic acquisition appears to be a reasonable strategy because systole has a stable length of approximately $200 \mathrm{~ms}$, is less sensitive to RR variability and arrhyth$\mathrm{mia}$, and is less prone to artifacts due to its thicker myocardium [26]. In addition, the thicker myocardium allows easier contour delineation and evaluation of transmural contrast enhancement.

\section{Wide-detector coverage}

In order to obtain whole heart dynamic CTP with high sampling rate of each heartbeat, wide-detector CT scanners with zaxis coverage of $16 \mathrm{~cm}$ is essential. Although (sub-)total coverage of left-ventricular myocardium is feasible by narrower detector scanners with shuttle mode, the sampling rate for a given part of myocardium is not high: every second heart beat for a heart rate of 63 beats per minute or less and every forth heart beat for a heart rate greater than 63 beats per minute $[26,27]$. Moreover, combination of temporally different cranial and caudal parts can potentially associate image artifacts along the border of the 2 parts. In these regards, area-detector CTs have distinct advantages over dual-source CTs. An additional advantage of wider-detector $\mathrm{CT}$ is the ability to acquire CCTA during the dynamic scan [23]. On the other hand, the capability for wide $\mathrm{z}$-axis dynamic imaging of every heart beat can easily lead to excessive radiation. Every dose reduction strategy such as low-tube-voltage setting, tube current modulation, and reduction of dynamic phases should be considered to avoid unneeded radiation exposure.

Table 1. Characteristics of computer tomography scanners capable of dynamic CTP with wide z-axis coverage

\begin{tabular}{clcccc}
\hline \multirow{2}{*}{ Manufacturer } & \multicolumn{1}{c}{ Model } & Number of detector-rows & $\begin{array}{c}\text { Z-axis coverage } \\
\text { of dynamic CTP }(\mathrm{mm})\end{array}$ & $\begin{array}{c}\text { Rotation speed } \\
(\mathrm{ms})\end{array}$ & $\begin{array}{c}\text { Half scan temporal } \\
\text { resolution (ms) }\end{array}$ \\
\hline Canon & GENESIS/ViSION & 320 & 160 & 275 & 138 \\
GE & Revolution & 256 & 160 & 280 & 140 \\
Philips & iCT & 128 & 78 & 270 & 135 \\
Siemens & Force & 96 & 105 (shuttle mode) & 250 & 66 (dual-source) \\
Siemens & Drive/ Flash & 64 & 73 (shuttle mode) & 280 & 75 (dual-source) \\
\hline
\end{tabular}

CTP: computed tomography perfusion 


\section{Beam hardening correction and noise reduction}

The beam-hardening effect is caused by preferential absorption of low-energy photons as a polychromatic X-ray beam passes through an object. The resulting filtered beam with higher mean energy can result in so-called beam hardening artifacts typically recognized as low-attenuation between high attenuation structures. Conventional beam-hardening correction algorithms used in body CT imaging have been specifically optimized to correct beam-hardening artifact from bone, which is the most substantial high-attenuation source of beam hardening. For accurate assessment of myocardial perfusion, beam hardening effects from temporally variable high attenuation in heart chambers and great vessels caused by iodine contrast material also need to be managed [28]. In dynamic CTP, the timedependent iodine distribution and its resulting beam hardening effect can be calculated by analyzing the temporal voxel changes, thus distinguishing from beam hardening effects from other high-attenuation materials such as bone $[29,30]$. This type of spatio-temporal analysis also greatly contributes to noise reduction (and eventually dose reduction) of dynamic CTP [25,29,30] and may be combined with iterative reconstruction algorithms $[31,32]$.

\section{QUANTITATIVE MYOCARDIAL PERFUSION ESTIMATION}

The most important advantage of dynamic CTP over static CTP is the feasibility of full quantification of myocardial perfusion in $\mathrm{mL} / \mathrm{min} / \mathrm{g}$. Although there are a number of approaches for full quantification [33,34], the maximum slope model has been used in most previous patient studies with only a few exceptions (model-based deconvolution [24] and single-tissue compartment model [23]). In the maximum slope method, myocardial blood flow (MBF) is estimated as the ratio of the maximum instantaneous slope of the myocardial iodine enhancement curve to the maximum value of the input function, multiplied by the reciprocal of the tissue density based on the assumption that the rate of arrival of contrast agent is proportional to the tissue perfusion level [34]. However, mathematically, MBF determined by the equation is equal to the blood-to-myocardium transfer constant, Ktrans in the Patlak model, which requires flow-dependent extraction fraction of contrast medium to be converted to MBF $[35,36]$. In addition, limited sampling rate may lead to substantial underestimation of MBF [35]. Expected systemic underestimation of MBF by the maximum slope method has been seen experimentally in comparison with microsphere-derived MBF $[37,38]$. Nonetheless, it has been demonstrated in porcine animal models that CT-derived MBF based on the maximum slope method shows good correlation with coronary artery blood flow and fractional flow reserve (FFR) $[39,40]$ and permits more accurate demonstration of the hemodynamic effect of moderate (50\%) coronary stenosis compared to measurement of myocardial enhancement at single-phase high-pitch acquisition [41]. In our experience, quantitative analysis is more sensitive for hypoperfusion than visual assessment of dynamic $\mathrm{CT}$ image datasets (Fig. 1) and improves inter-observer agreement for detecting significant CAD. Further investigation is warranted for reliability of MBF quantification by CT regarding its test-retest reproducibility and correlation with ${ }^{15} \mathrm{O}$-water PET, which is the established clinical standard for MBF quantification. Furthermore, lack of standardized acquisition and modeling protocols for MBF quantification complicates the generalization of study results obtained using a different CT protocol and/or mathematical modeling.

\section{DIAGNOSTIC PERFORMANCE OF DYNAMIC CTP}

Diagnostic performance of dynamic CTP has been validated against different reference standards including MRI [42-45], SPECT $[43,46,47]$, and coronary angiography with FFR measurement [21,27,48-53]. Available data are scarce on the diagnostic performance of dynamic CTP for detecting perfusion defects determined by MRI or SPECT. However, reported segment-based sensitivity and specificity of dynamic CTP are 78$81 \%$ and $75-94 \%$, respectively, when MRI is used as the reference $[44,45]$. These values are $83-85 \%$ and $78-92 \%$, respectively, in comparison with SPECT [46,47]. So far, there are 8 single center studies evaluating dynamic CTP with FFR as the reference standard (Table 2). The sensitivity, specificity, PPV, and NPV range from $73-98 \%, 68-100 \%, 49-100 \%$, and $74-98 \%$, respectively, with pooled sensitivity of $84 \%$ and pooled specificity of $80 \%$. It has been demonstrated that specificity and PPV of CCTA can be improved with quantitative dynamic CTP $[45,50]$.

In all studies except one by Huber et al. [21], dual-source CT and its commercially available perfusion quantification software were used for the evaluation of diagnostic accuracy. As summarized in Table 2, in spite of the use of the same CT technology and mathematical algorithm and relatively small variation in the mean MBF values in ischemic myocardium $(70-79 \mathrm{~mL} /$ $100 \mathrm{~mL} / \mathrm{min}$ ), the best cut-off MBF value for distinguishing ischemic and remote myocardium varies from 75 to $103 \mathrm{~mL} / 100$ $\mathrm{mL} / \mathrm{min}$. This wide range of cut-off values may be related to differences in study design, patient risk profile, prevalence of CAD, and FFR threshold. In addition, hyperemic MBF in remote myocardium can be influenced by other factors such as age, gender, race, $\mathrm{BMI}$, insulin resistance, arteriolar response to vasodilators, and presence of collateral vessels [54-57]. Debatably, relative MBF normalized by remote MBF may provide more accurate identification of hemodynamically significant CAD than abso- 

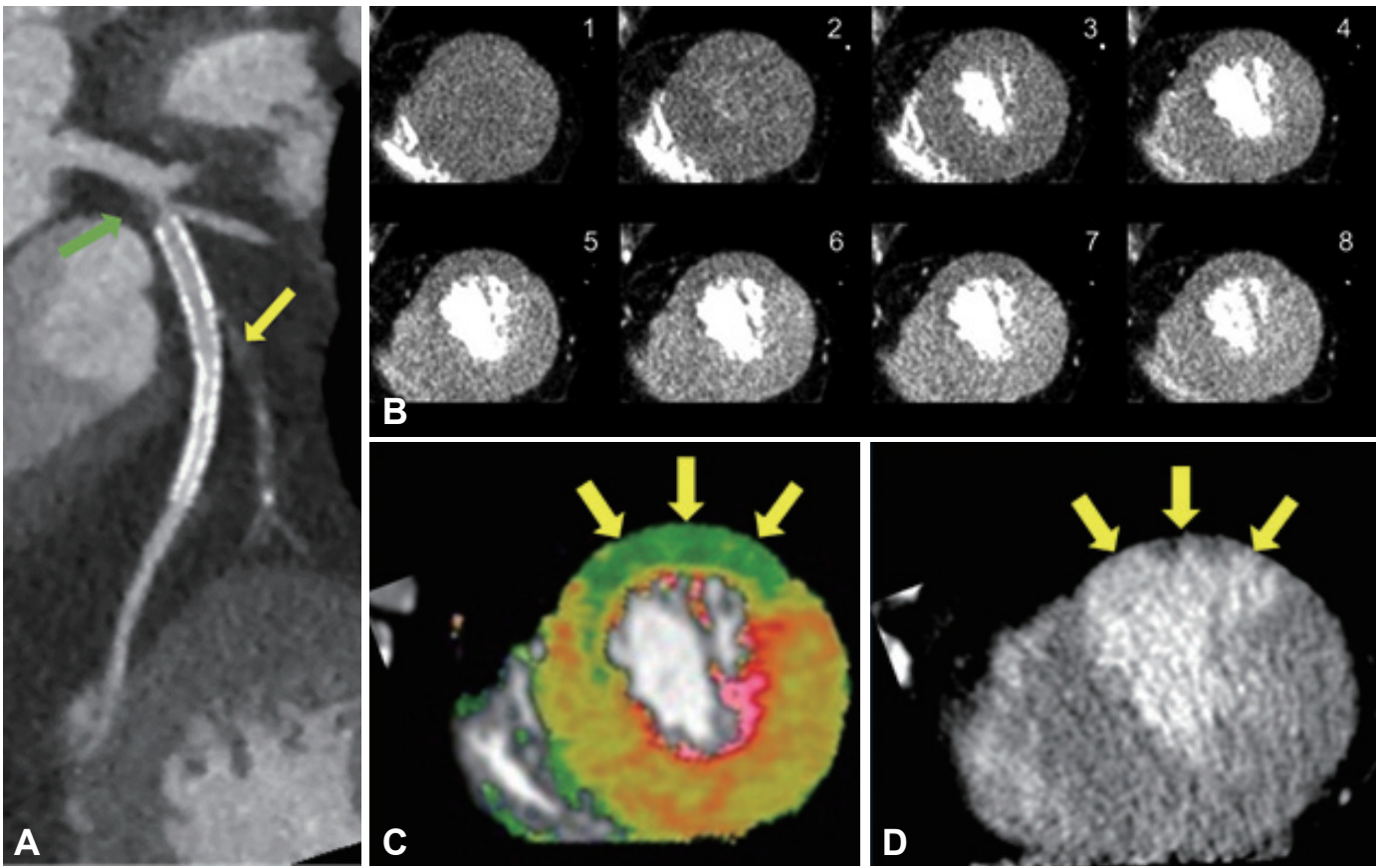

Fig. 1. A 58-year-old male with stenting to the LAD artery. Coronary CT angiography demonstrated moderate stenosis in the proximal $L A D$ (green arrow) and an occluded diagonal branch (yellow arrow) (A). Reduced perfusion was hardly recognized on CT perfusion images of 8 continuous dynamic phases (B) but was evident in the anterior wall (arrows) subtended by the occluded diagonal branch on the myocardial blood flow map (C). Delayed enhancement CT revealed presence of infarcted myocardium in the area (D). LAD: left anterior descending, CT: computed tomography.

Table 2. Vessel-based diagnostic accuracy of dynamic CT perfusion compared with invasive coronary angiography with fractional flow reserve for the diagnosis of hemodynamically significant coronary artery disease

\begin{tabular}{|c|c|c|c|c|c|c|c|c|c|c|}
\hline Author & $\mathrm{N}$ & CT scanner & $\begin{array}{l}\text { SE } \\
(\%)\end{array}$ & $\begin{array}{l}\text { SP } \\
(\%)\end{array}$ & $\begin{array}{l}\text { PPV } \\
(\%)\end{array}$ & $\begin{array}{c}\text { NPV } \\
(\%)\end{array}$ & $\begin{array}{l}\text { MBF stress } \\
\text { remote }\end{array}$ & $\begin{array}{c}\text { MBF stress } \\
\text { ischemic }\end{array}$ & $\begin{array}{l}\text { Best MBF } \\
\text { cut-off }\end{array}$ & $\begin{array}{c}\text { Radiation dose } \\
(\mathrm{mSv})\end{array}$ \\
\hline Bamberg et al. [27] & 33 & Flash & 93 & 87 & 75 & 97 & $105 \pm 34$ & $73 \pm 26$ & 75 & $10.0^{\dagger}$ \\
\hline Greif et al. [48] & 65 & Flash & 95 & 74 & 49 & 98 & $123 \pm 34$ & $79 \pm 26$ & 75 & $9.7^{\dagger}$ \\
\hline Huber et al. [21] & 32 & iCT & 76 & 100 & 100 & 91 & $310 \pm 130$ & $150 \pm 160$ & 164 & $9.5^{\dagger}$ \\
\hline Rossi et al. [50] & 80 & Flash & 88 & 90 & 77 & 95 & $100(83-119)$ & $70(62-76)$ & 78 & $9.4^{\ddagger}$ \\
\hline Kono et al. [49] & 42 & Flash & 98 & 70 & 76 & 97 & $116 \pm 27$ & $76 \pm 23$ & $103^{*}$ & $9.4^{\ddagger}$ \\
\hline Goto et al. [53] & 51 & Flash & 84 & 84 & 82 & 86 & $99 \pm 36$ & $74 \pm 27$ & $89 *$ & $5.3^{\ddagger}$ \\
\hline Coenen et al. [52] & 43 & $\begin{array}{l}\text { Flash } \\
\text { Force }\end{array}$ & 75 & 78 & 78 & 75 & $92 \pm 23$ & $71 \pm 24$ & 76 & $9.0^{\ddagger}$ \\
\hline Coenen et al. [51] & 74 & $\begin{array}{l}\text { Flash } \\
\text { Force }\end{array}$ & 73 & 68 & 67 & 74 & 108 & 79 & 91 & $9.3^{\ddagger}$ \\
\hline Pooled & 420 & & 84 & 80 & 72 & 88 & 106.5 (median) & 75 (median) & 83.5 (median) & $8.4^{\ddagger}$ \\
\hline
\end{tabular}

$\mathrm{MBF}$ was presented in $\mathrm{mL} / 100 \mathrm{~mL} / \mathrm{min}$. *best diagnostic performance was achieved based on relative MBF cut-off (Kono et al. [49] MBF ratio of 0.85; Goto et al. [53] transmural perfusion ratio of 0.899$),{ }^{\dagger}$ conversion factor $=0.017,{ }^{\ddagger}$ conversion factor $=0.014$. N: number of patients, MBF: myocardial blood flow, NPV: negative predictive value, PPV: positive predictive value, SE: sensitivity, SP: specificity

lute MBF $[49,53]$. We should also recognize that FFR is a measure of lesion specific hemodynamic significance of stenosis, not a measure of myocardial perfusion, which is governed by not only epicardial coronary stenosis, but also microvascular environment and collateral circulation. In order to enable accurate interpretation of quantitative MBF values, a large database on normal values including physiological regional heterogeneity and establishment of the prognostic implication of MBF value are arguably necessary. Only limited data are available on normal MBF values at this point $[58,59]$ (Table 3 ).

\section{RADIATION OF DYNAMIC CTP}

Radiation exposure associated with dynamic CTP remains a concern. The average effective radiation dose ranges between $5.3 \mathrm{mSv}$ to $10.0 \mathrm{mSv}$ in the previous reports listed in Table 3. 
Dynamic CT Perfusion Imaging

Table 3. MBF values in normal volunteers and low risk subjects

\begin{tabular}{llclcccc}
\hline \multicolumn{1}{c}{ Author } & N & CT scanner & \multicolumn{1}{c}{ Subjects } & Age (yr) & MBF stress & MBF rest & Perfusion reserve \\
\hline Kim et al. [58] & 19 & Flash & Healthy volunteers & $33-60$ & $173 \pm 33$ & $83 \pm 21$ & $2.20 \pm 0.53$ \\
Ho et al. [59] & 35 & Flash & Low-risk individuals & $25-64$ & $135 \pm 29$ & $74 \pm 16$ & $1.86 \pm 0.38$
\end{tabular}

MBF was presented in $\mathrm{mL} / 100 \mathrm{~mL} / \mathrm{min}$. N: number of patients, MBF: myocardial blood flow, CT: computed tomography

However, use of a low tube voltage protocol $(80 \mathrm{kV} / 379 \mathrm{~mA})$ instead of a conventional protocol (100 kV/300 mAs) enables $40 \%$ dose reduction without affecting image quality or $\mathrm{MBF}$ quantification in patients with normal body mass index [60]. In addition, use of an automatic exposure control system could substantially reduce radiation dose [61]. Dynamic CTP with 3.5 $\mathrm{mSv}$ has been reported with a combined use of $70 \mathrm{kVp}$ and an automatic exposure control system [62].

\section{IMPLEMENTATION OF STRESS DYNAMIC CTP IN COMPREHENSIVE CARDIAC CT}

In clinical practice, stress dynamic CTP is often implemented as a part of a comprehensive cardiac CT protocol together with other modules such as CCTA, rest dynamic CTP, and CT delayed enhancement.

\section{CCTA}

CCTA is generally considered an essential module in a comprehensive cardiac CT. Most reports on CTP perform CCTA and CTP within a single imaging session. However, considering the recent wide-spread use of CCTA in clinical practice, once established as an accurate myocardial perfusion imaging, CTP may be performed independently as a second-line test when CCTA shows CAD of uncertain functional significance or is non-diagnostic. With a wide-area detector with $16 \mathrm{~cm} \mathrm{z}$-axis coverage, CCTA may be acquired during rest dynamic CTP [23].

\section{Rest dynamic CTP}

Rest dynamic CTP has been performed in few limited research environments $[23,58,59,63]$. This is simply because CCTA has higher priority than quantitative perfusion information at resting state. Although rest dynamic CTP allows calculation of coronary flow reserve (CFR), PET studies have shown that hyperemic MBF alone may perform equally as well as CFR for detecting coronary stenosis $[64,65]$. Having said that, a new study has suggested the prognostic value of integrated assessment of hyperemic MBF and CFR [66]. In a clinical scenario where dynamic CTP is used as a second-line test, performing both stress and rest dynamic CTP may be an attractive strategy.

\section{Delayed enhancement}

CT delayed enhancement (CTDE) shares the same pathophysiological principle with delayed enhancement MRI, allowing

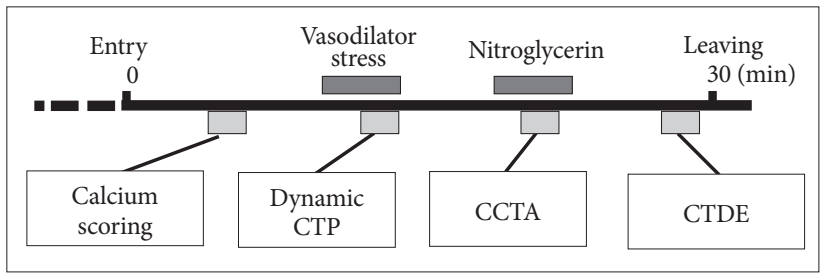

Fig. 2. Image acquisition timeline of a comprehensive cardiac CT study. Calcium scoring, stress dynamic CTP, rest CCTA, and CTDE can be performed in 30 minutes. CT: computed tomography, CTP: CT perfusion, CCTA: coronary CT angiography, CTDE: CT delayed enhancement.

infarct detection and viability assessment. Side-by-side comparison of stress CTP and CTDE is useful for accurate differentiation between ischemic and infarcted myocardium. Unfortunately, CTDE is not widely used due to low contrast-to-noise ratio; however, its quality has been significantly improved with the recent advancement in image acquisition, reconstruction, and postprocessing [67-69]. Assessment of myocardial delayed enhancement appears to be especially important in the delineation of ischemic but viable myocardium in patients with history of myocardial infarction and/or prior stent implantation [53]. Moreover, CTDE may be used to estimate extracellular volume fraction of the left ventricular myocardium [70,71].

\section{Stress CTP first vs. CCTA first}

Stress CTP first, followed by CCTA is preferred for the detection of myocardial ischemia because the stress scan will produce "clean," uncontaminated data. After the stress scan, beta blockers and sublingual nitroglycerin can be administered to optimize CCTA. A timeline of a comprehensive cardiac CT protocol is presented in Fig. 2. CCTA first, followed by stress CTP may appear clinically reasonable since stress CTP can be avoided when obstructive CAD is excluded by CCTA. However, this is not true because of the difficulty of assessing CCTA and deciding whether or not to proceed to stress CTP. Moreover, the time slot required for CCTA becomes unpredictable, which causes substantial inconvenience in scheduling of CT scans. From a technical perspective, cross-contamination of contrast in the stress perfusion scan and beta blockers used to optimize CCTA may mask ischemia. In addition, refrainment from caffeine 24 hours before CT may not be achieved thoroughly in this approach due to the optional nature of the stress scan. 


\section{REAL-WORLD INTERPRETATION OF DYNAMIC CTP}

Stress myocardial perfusion can be considered normal when uniformly high (i.e., $>110 \mathrm{~mL} / 100 \mathrm{~mL} / \mathrm{min}$ for dual-source CT and maximum slope method) MBF is demonstrated (Fig. 3). Uniformly low MBF ( $<75 \mathrm{~mL} / 100 \mathrm{~mL} / \mathrm{min}$ ) is rarely seen, but is most likely due to no or insufficient hyperemic response to vasodilators, which may hamper ischemia detection (Fig. 4). Once sufficient hyperemia is confirmed by high MBF in remote myocardium, hemodynamic significance of coronary artery stenosis may be assessed based on absolute $\mathrm{MBF}$ value in the vessel territory (Fig. 5). However, as discussed earlier, a universally applicable MBF threshold for myocardial ischemia may not exist since normal MBF varies from patient to patient. Therefore, it is important to consider relative MBF values in the final judgement of ischemia (Fig. 6). Moreover, reduction of perfusion may be observed in the absence of obstructive CAD as a result of microvascular dysfunction (Fig. 7). Assessment of hemodynamic significance of epicardial coronary stenosis is a challenge in the presence of concomitant microvascular dysfunction.

\section{CONCLUSIONS}

Dynamic CTP is a novel myocardial perfusion imaging technique that may open the era of routine absolute quantification of MBF. However, it is a still-nascent technology, and standard-
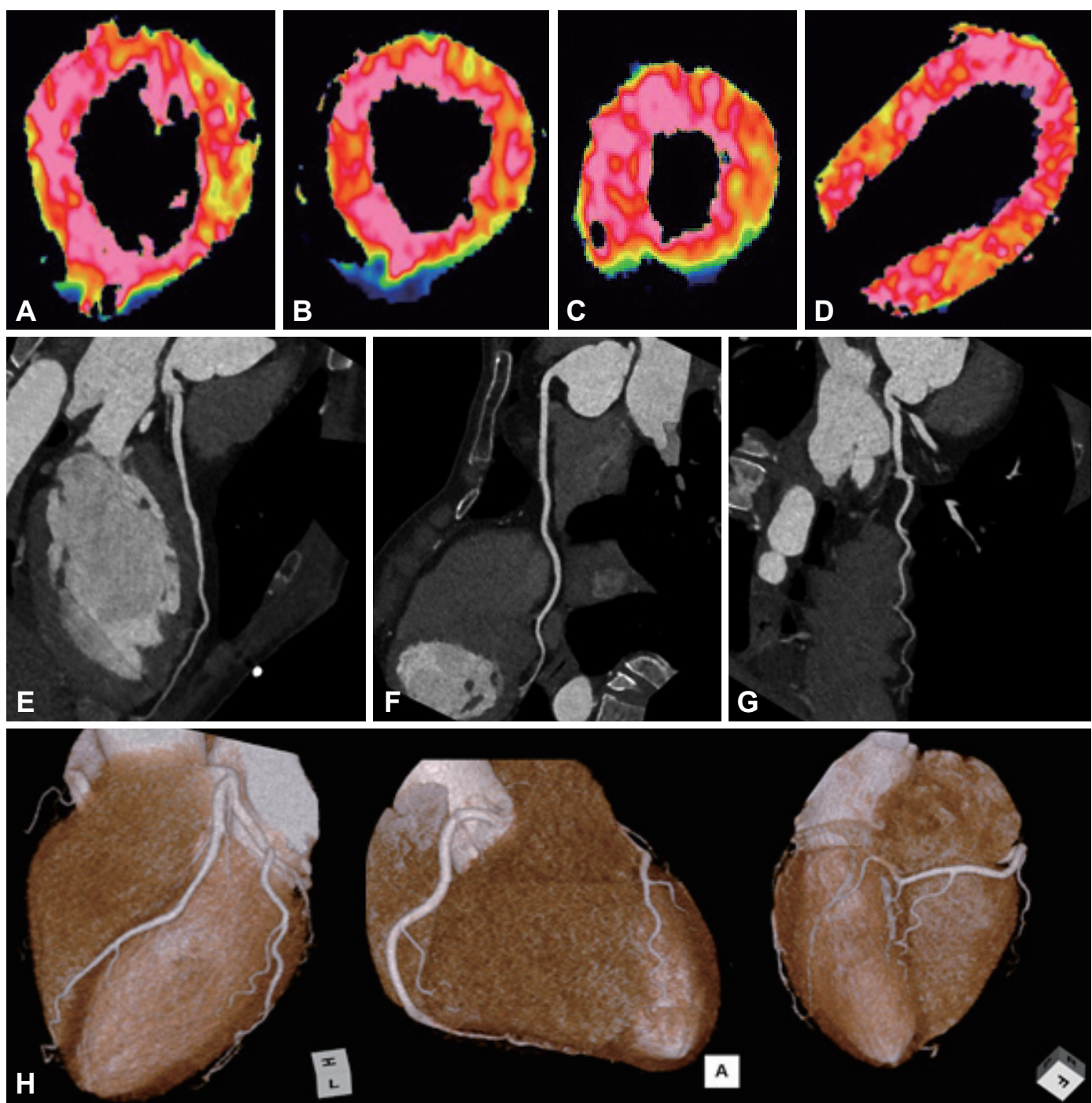

Fig. 3. Basal, mid, and apical short axis (A, B, and C) and horizontal long axis (D) stress dynamic computed tomography-derived MBF maps of the left ventricle with a color-coded overlay showing uniformly normal absolute MBF values $(200-230 \mathrm{~mL} / 100 \mathrm{~mL} / \mathrm{min})$ in a 54-year-old female with suspected coronary artery disease. Curved multiplanar reformatted images of the left anterior descending (E), right coronary artery $(F)$, and left circumflex artery $(G)$ and three-dimensional volume rendered images $(H)$, all show normal coronary arteries. MBF: myocardial blood flow. 

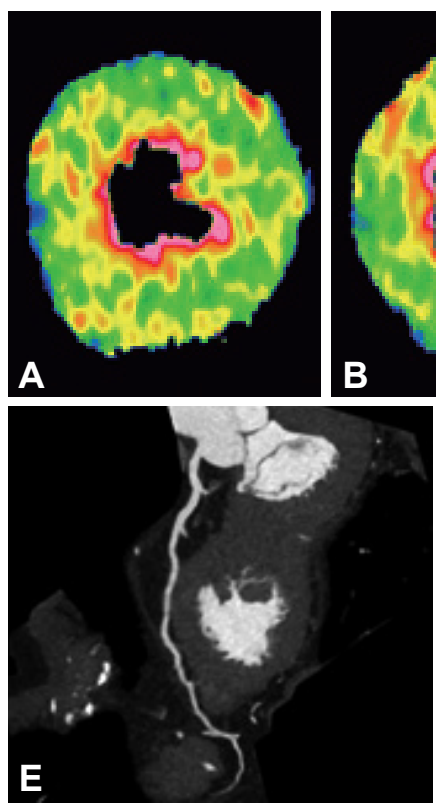
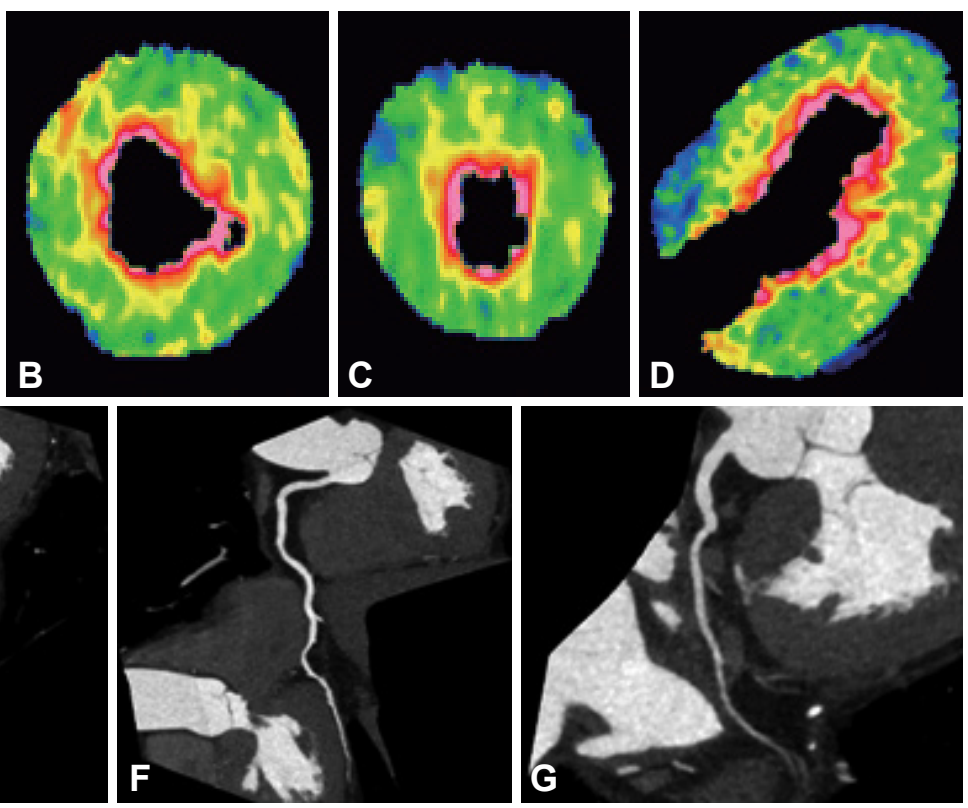

Fig. 4. Basal, mid, and apical short axis (A, B, and C) and horizontal long axis (D) stress dynamic computed tomography-derived MBF maps of the left ventricle with a color-coded overlay in a 70-year-old male with suspected coronary artery disease showing uniformly reduced absolute MBF values $(70-80 \mathrm{~mL} / 100 \mathrm{~mL} / \mathrm{min})$. Curved multiplanar reformatted images of the left anterior descending (E), right coronary artery $(F)$, and left circumflex artery $(G)$ show no significant coronary artery stenosis. MBF: myocardial blood flow.
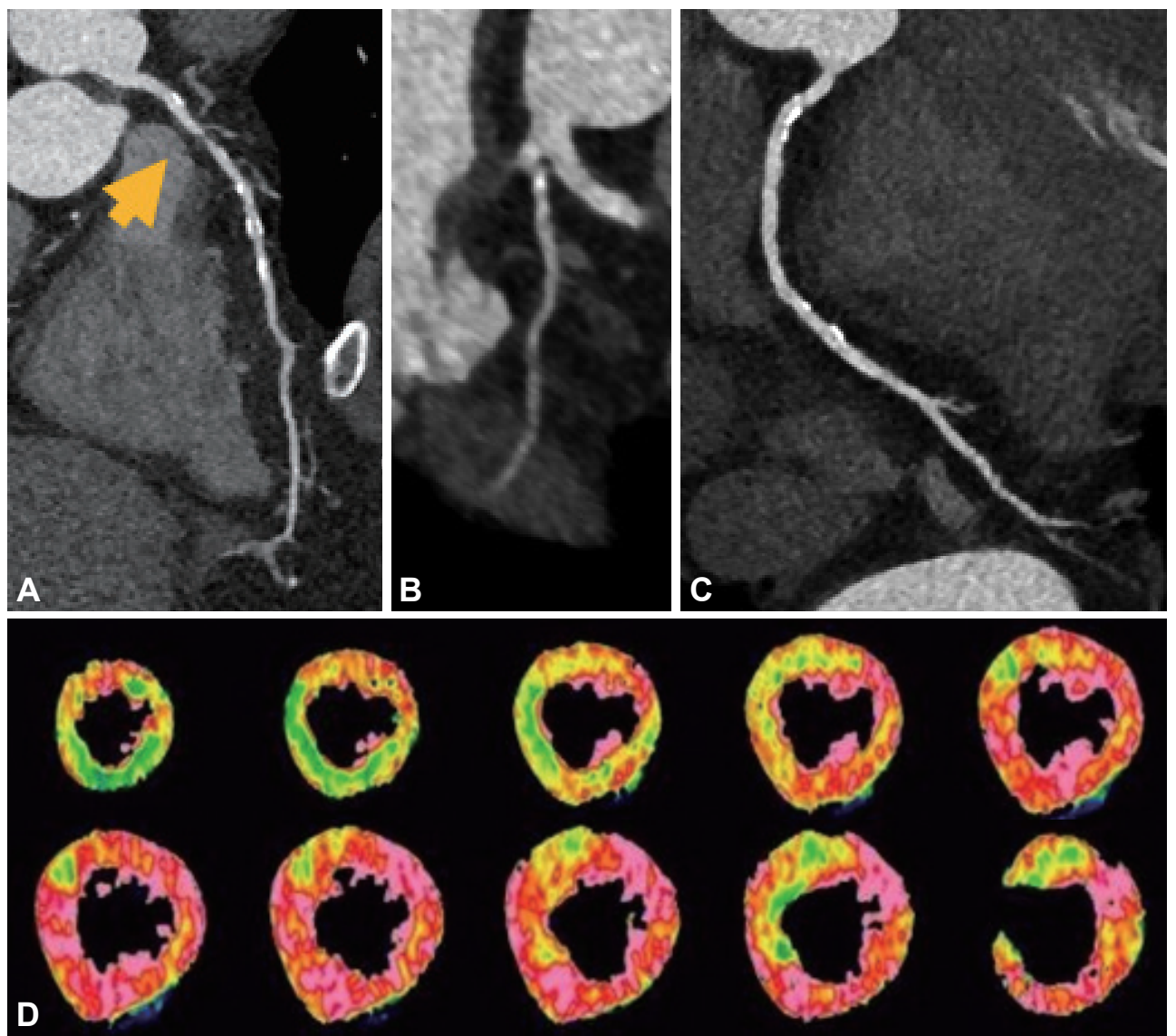

Fig. 5. In a 72-year-old female with dyslipidemia and diabetes who presented with electrocardiogram abnormality, coronary computed tomography angiography demonstrated severe stenosis (yellow arrow) in the left descending artery (A) and no significant stenosis in the left circumflex artery (B) or right coronary artery (C). Dynamic computer tomography perfusion images in the short axis (D) showed reduced myocardial blood flow in the left descending artery territory. 

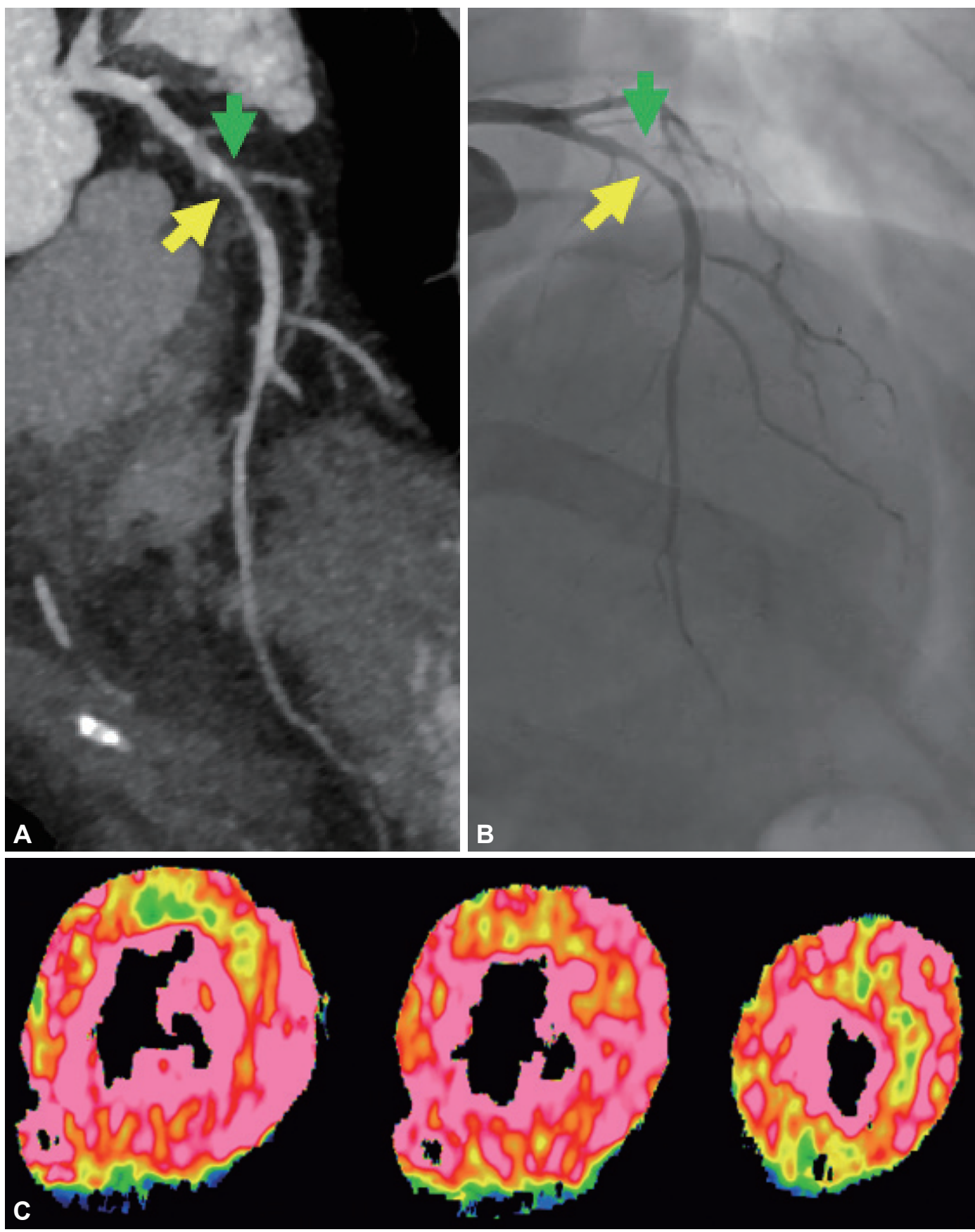

Fig. 6. In a 52-year-old female with hypertension, dyslipidemia, and diabetes who presented with atypical chest pain, coronary CT angiography $(A)$ and invasive coronary angiography $(B)$ demonstrated moderate stenosis in the proximal left descending artery (yellow arrow) and severe stenosis in the diagonal branch (green arrow). Dynamic CT perfusion images in the short axis (C) showed MBF values of 100-110 $\mathrm{mL} / 100 \mathrm{~mL} / \mathrm{min}$ in the diagonal branch territory, which were substantially lower compared with remote segments $(170-180 \mathrm{~mL} / 100 \mathrm{~mL} / \mathrm{min})$. Although absolute MBF in the diagonal branch territory was well above thresholds of myocardial ischemia $(75-103 \mathrm{~mL} / 100 \mathrm{~mL} / \mathrm{min})$ in the literature, concordance of stenosis and relative reduction of MBF suggested its hemodynamic significance. CT: computed tomography, MBF: myocardial blood flow.

ization of acquisition method, mathematical modeling, and interpretation remain to be achieved. Nonetheless, high diagnostic values of dynamic CTP are seen for the detection of myocardial ischemia determined by FFR. In addition, cardiac CT has the advantage of providing integrated information of morphology and function in a single examination. Large-scale clinical trials are warranted to further evaluate its accuracy, clinical role, and cost effectiveness. 

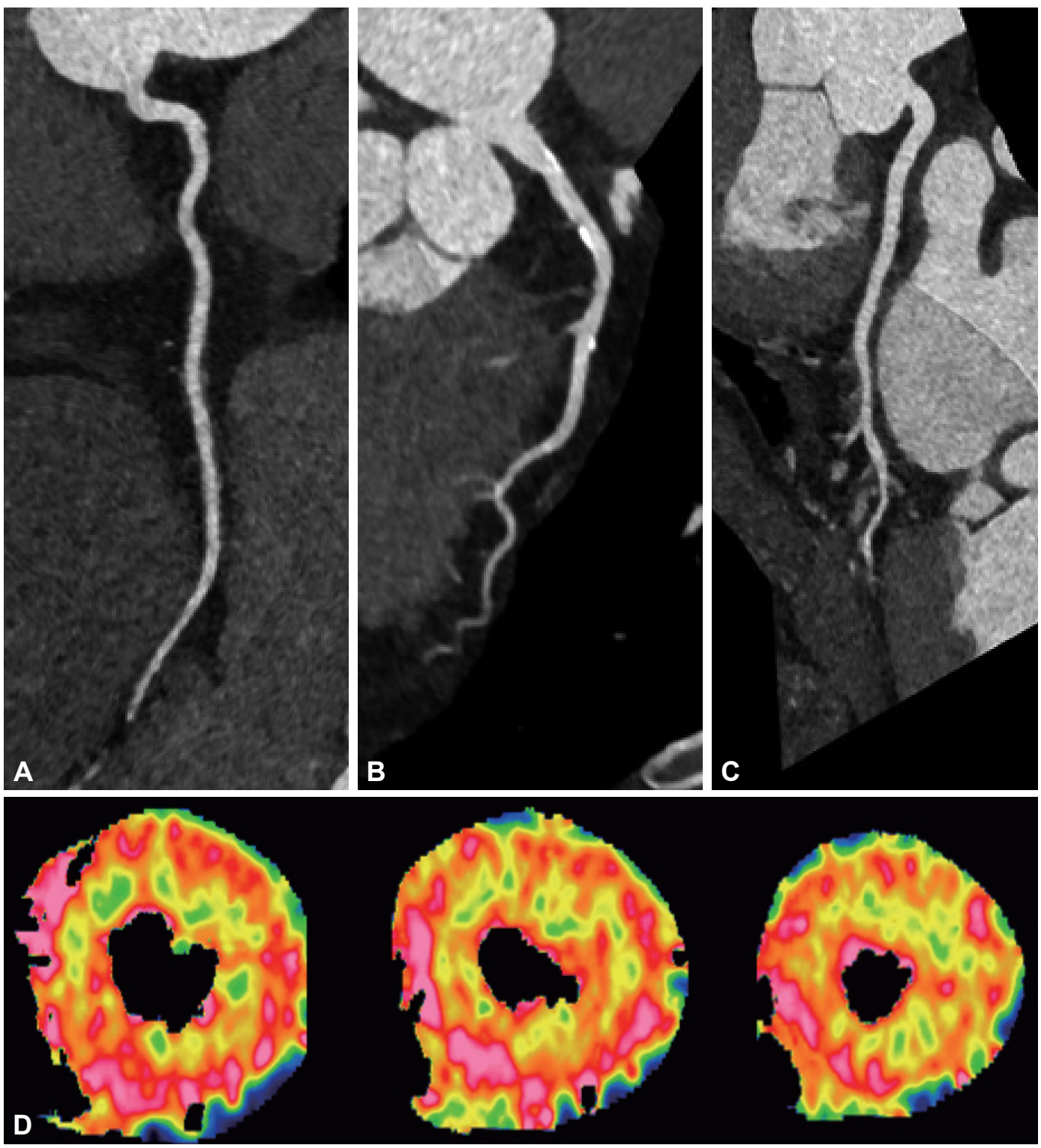

Fig. 7. This figure shows images of an 85 -year-old male with hypertension. On coronary CT angiography, only mild calcified plaque was present in the left descending artery (B), and no plaque or stenosis was detected in the right coronary artery (A) or the left circumflex artery (C). Left ventricular hypertrophy was not present (not shown). Dynamic CT perfusion images demonstrated sub-endocardial reduced perfusion diffusely distributed from base to apex (D). This stress perfusion finding was attributed to microvascular dysfunction in the absence of obstructive stenosis in the epicardial coronary arteries. CT: computed tomography.

\section{Conflicts of Interest}

The authors declare that they have no conflict of interest.

\section{REFERENCES}

1. Marwick TH, Cho I, O Hartaigh B, Min JK. Finding the gatekeeper to the cardiac catheterization laboratory: coronary CT angiography or stress testing? J Am Coll Cardiol 2015;65:2747-2756.

2. SCOT-HEART investigators. CT coronary angiography in patients with suspected angina due to coronary heart disease (SCOT-HEART): an openlabel, parallel-group, multicentre trial. Lancet 2015;385:2383-2391.

3. Williams MC, Hunter A, Shah ASV, Assi V, Lewis S, Smith J, et al. Use of coronary computed tomographic angiography to guide management of patients with coronary disease. J Am Coll Cardiol 2016;67:1759-1768.

4. Adamson PD, Hunter A, Williams MC, Shah AS, McAllister DA, Pawade
TA, et al. Diagnostic and prognostic benefits of computed tomography coronary angiography using the 2016 National Institute for Health and Care Excellence guidance within a randomised trial. Heart 2018;104:207214.

5. Blankstein R, Di Carli MF. Integration of coronary anatomy and myocardial perfusion imaging. Nat Rev Cardiol 2010;7:226-236.

6. Boden WE, O’Rourke RA, Teo KK, Hartigan PM, Maron DJ, Kostuk WJ, et al.; COURAGE Trial Research Group. Optimal medical therapy with or without PCI for stable coronary disease. N Engl J Med 2007;356:1503-1516.

7. De Bruyne B, Pijls NH, Kalesan B, Barbato E, Tonino PA, Piroth Z, et al.; FAME 2 Trial Investigators. Fractional flow reserve-guided PCI versus medical therapy in stable coronary disease. N Engl J Med 2012;367:9911001.

8. Tonino PA, Fearon WF, De Bruyne B, Oldroyd KG, Leesar MA, Ver Lee $\mathrm{PN}$, et al. Angiographic versus functional severity of coronary artery ste- 
noses in the FAME study fractional flow reserve versus angiography in multivessel evaluation. J Am Coll Cardiol 2010;55:2816-2821.

9. Schuijf JD, Ko BS, Di Carli MF, Hislop-Jambrich J, Ihdayhid AR, Seneviratne SK, et al. Fractional flow reserve and myocardial perfusion by computed tomography: a guide to clinical application. Eur Heart J Cardiovasc Imaging 2018;19:127-135.

10. Kurata A, Mochizuki T, Koyama Y, Haraikawa T, Suzuki J, Shigematsu Y, et al. Myocardial perfusion imaging using adenosine triphosphate stress multi-slice spiral computed tomography: alternative to stress myocardial perfusion scintigraphy. Circ J 2005;69:550-557.

11. Bischoff B, Bamberg F, Marcus R, Schwarz F, Becker HC, Becker A, et al. Optimal timing for first-pass stress CT myocardial perfusion imaging. Int J Cardiovasc Imaging 2013;29:435-442.

12. Otton J, Morton G, Schuster A, Bigalke B, Marano R, Olivotti L, et al. A direct comparison of the sensitivity of $\mathrm{CT}$ and $\mathrm{MR}$ cardiac perfusion using a myocardial perfusion phantom. J Cardiovasc Comput Tomogr 2013;7:117124.

13. Pelgrim GJ, Nieuwenhuis ER, Duguay TM, van der Geest RJ, VargaSzemes A, Slump CH, et al. Optimal timing of image acquisition for arterial first pass CT myocardial perfusion imaging. Eur J Radiol 2017;86:227233.

14. Tanabe Y, Kido T, Kurata A, Uetani T, Fukuyama N, Yokoi T, et al. Optimal scan time for single-phase myocardial computed tomography perfusion to detect myocardial ischemia. Derivation cohort from dynamic myocardial computed tomography perfusion. Circ J 2016;80:2506-2512.

15. Yang DH, Kim YH, Roh JH, Kang JW, Han D, Jung J, et al. Stress myocardial perfusion CT in patients suspected of having coronary artery disease: visual and quantitative analysis-validation by using fractional flow reserve. Radiology 2015;276:715-723.

16. van Rosendael AR, de Graaf MA, Dimitriu-Leen AC, van Zwet EW, van den Hoogen IJ, Kharbanda RK, et al. The influence of clinical and acquisition parameters on the interpretability of adenosine stress myocardial computed tomography perfusion. Eur Heart J Cardiovasc Imaging 2017; 18:203-211.

17. Abbara S, Blanke P, Maroules CD, Cheezum M, Choi AD, Han BK, et al. SCCT guidelines for the performance and acquisition of coronary computed tomographic angiography: a report of the society of Cardiovascular Computed Tomography Guidelines Committee: Endorsed by the North American Society for Cardiovascular Imaging (NASCI). J Cardiovasc Comput Tomogr 2016;10:435-449.

18. Primak AN, Dong Y, Dzyubak OP, Jorgensen SM, McCollough CH, Ritman EL. A technical solution to avoid partial scan artifacts in cardiac MDCT. Med Phys 2007;34:4726-4737.

19. Ramirez-Giraldo JC, Yu L, Kantor B, Ritman EL, McCollough CH. A strategy to decrease partial scan reconstruction artifacts in myocardial perfusion CT: phantom and in vivo evaluation. Med Phys 2012;39:214223.

20. Stenner P, Schmidt B, Bruder H, Allmendinger T, Haberland U, Flohr T, et al. Partial scan artifact reduction (PSAR) for the assessment of cardiac perfusion in dynamic phase-correlated CT. Med Phys 2009;36:5683-5694.

21. Huber AM, Leber V, Gramer BM, Muenzel D, Leber A, Rieber J, et al. Myocardium: dynamic versus single-shot CT perfusion imaging. Radiology 2013;269:378-386.

22. Bruder H, Raupach R, Klotz E, Stierstorfer K, Flohr T. Spatio-temporal filtration of dynamic CT data using diffusion filters. Proceedings of SPIE Medical Imaging; 2009 Feb 7-12; Lake Buena Vista (Orlando Area), Florida, United States. Proc SPIE 7258, Medical Imaging 2009: Physics of Medical Imaging.725857.

23. Kikuchi Y, Oyama-Manabe N, Naya M, Manabe O, Tomiyama Y, Sasaki T, et al. Quantification of myocardial blood flow using dynamic 320-row multi-detector CT as compared with 15O-H2O PET. Eur Radiol 2014;24: 1547-1556.

24. Kurata A, Kawaguchi N, Kido T, Inoue K, Suzuki J, Ogimoto A, et al. Qualitative and quantitative assessment of adenosine triphosphate stress whole-heart dynamic myocardial perfusion imaging using 256-slice com- puted tomography. PLoS One 2013;8:e83950.

25. Muenzel D, Kabus S, Gramer B, Leber V, Vembar M, Schmitt H, et al. Dynamic CT perfusion imaging of the myocardium: a technical note on improvement of image quality. PLoS One 2013;8:e75263.

26. Mahnken AH, Klotz E, Pietsch H, Schmidt B, Allmendinger T, Haberland $\mathrm{U}$, et al. Quantitative whole heart stress perfusion CT imaging as noninvasive assessment of hemodynamics in coronary artery stenosis: preliminary animal experience. Invest Radiol 2010;45:298-305.

27. Bamberg F, Becker A, Schwarz F, Marcus RP, Greif M, von Ziegler F, et al. Detection of hemodynamically significant coronary artery stenosis: incremental diagnostic value of dynamic CT-based myocardial perfusion imaging. Radiology 2011;260:689-698.

28. Kitagawa K, George RT, Arbab-Zadeh A, Lima JA, Lardo AC. Characterization and correction of beam-hardening artifacts during dynamic volume CT assessment of myocardial perfusion. Radiology 2010;256:111118.

29. Stenner P, Schmidt B, Allmendinger T, Flohr T, Kachelrie M. Dynamic iterative beam hardening correction (DIBHC) in myocardial perfusion imaging using contrast-enhanced computed tomography. Invest Radiol 2010;45:314-323.

30. So A, Imai Y, Nett B, Jackson J, Nett L, Hsieh J, et al. Technical note: evaluation of a 160-mm/256-row CT scanner for whole-heart quantitative myocardial perfusion imaging. Med Phys 2016;43:4821.

31. Tao Y, Chen GH, Hacker TA, Raval AN, Van Lysel MS, Speidel MA. Low dose dynamic CT myocardial perfusion imaging using a statistical iterative reconstruction method. Med Phys 2014;41:071914.

32. Gramer BM, Muenzel D, Leber V, von Thaden AK, Feussner H, Schnei$\operatorname{der} \mathrm{A}$, et al. Impact of iterative reconstruction on CNR and SNR in dynamic myocardial perfusion imaging in an animal model. Eur Radiol 2012;22:2654-2661.

33. Pelgrim GJ, Handayani A, Dijkstra H, Prakken NH, Slart RH, Oudkerk $\mathrm{M}$, et al. Quantitative myocardial perfusion with dynamic contrast-enhanced imaging in MRI and CT: theoretical models and current implementation. Biomed Res Int 2016;2016:1734190.

34. Bindschadler M, Modgil D, Branch KR, La Riviere PJ, Alessio AM. Comparison of blood flow models and acquisitions for quantitative myocardial perfusion estimation from dynamic CT. Phys Med Biol 2014;59:15331556.

35. Ishida M, Kitagawa K, Ichihara T, Natsume T, Nakayama R, Nagasawa N, et al. Underestimation of myocardial blood flow by dynamic perfusion CT: explanations by two-compartment model analysis and limited temporal sampling of dynamic CT. J Cardiovasc Comput Tomogr 2016;10:207-214.

36. Ishida M, Ichihara T, Nagata M, Ishida N, Takase S, Kurita T, et al. Quantification of myocardial blood flow using model based analysis of firstpass perfusion MRI: extraction fraction of Gd-DTPA varies with myocardial blood flow in human myocardium. Magn Reson Med 2011;66:13911399.

37. Bamberg F, Hinkel R, Schwarz F, Sandner TA, Baloch E, Marcus R, et al. Accuracy of dynamic computed tomography adenosine stress myocardial perfusion imaging in estimating myocardial blood flow at various degrees of coronary artery stenosis using a porcine animal model. Investigative Radiology 2012;47:71-77.

38. Pelgrim GJ, Das M, van Tuijl S, van Assen M, Prinzen FW, Stijnen M, et al. Validation of myocardial perfusion quantification by dynamic CT in an ex-vivo porcine heart model. Int J Cardiovasc Imaging 2017;33:18211830.

39. Rossi A, Uitterdijk A, Dijkshoorn M, Klotz E, Dharampal A, van Straten $\mathrm{M}$, et al. Quantification of myocardial blood flow by adenosine-stress CT perfusion imaging in pigs during various degrees of stenosis correlates well with coronary artery blood flow and fractional flow reserve. Eur Heart J Cardiovasc Imaging 2013;14:331-338.

40. Pelgrim GJ, Duguay TM, Stijnen JM, Varga-Szemes A, Van Tuijl S, Schoepf UJ, et al. Analysis of myocardial perfusion parameters in an ex-vivo porcine heart model using third generation dual-source CT. J Cardiovasc Comput Tomogr 2017;11:141-147. 
41. Schwarz F, Hinkel R, Baloch E, Marcus RP, Hildebrandt K, Sandner TA, et al. Myocardial CT perfusion imaging in a large animal model: comparison of dynamic versus single-phase acquisitions. JACC Cardiovasc Imaging 2013;6:1229-1238.

42. Bastarrika G, Ramos-Duran L, Rosenblum MA, Kang DK, Rowe GW, Schoepf UJ. Adenosine-stress dynamic myocardial CT perfusion imaging: initial clinical experience. Invest Radiol 2010;45:306-313.

43. Weininger M, Schoepf UJ, Ramachandra A, Fink C, Rowe GW, Costello P, et al. Adenosine-stress dynamic real-time myocardial perfusion $\mathrm{CT}$ and adenosine-stress first-pass dual-energy myocardial perfusion CT for the assessment of acute chest pain: initial results. Eur J Radiol 2012;81:37033710.

44. Kim SM, Choi JH, Chang SA, Choe YH. Detection of ischaemic myocardial lesions with coronary $\mathrm{CT}$ angiography and adenosine-stress dynamic perfusion imaging using a 128-slice dual-source CT: diagnostic performance in comparison with cardiac MRI. Br J Radiol 2013;86:20130481.

45. Bamberg F, Marcus RP, Becker A, Hildebrandt K, Bauner K, Schwarz F, et al. Dynamic myocardial CT perfusion imaging for evaluation of myocardial ischemia as determined by MR imaging. JACC Cardiovasc Imaging 2014;7:267-277.

46. Ho KT, Chua KC, Klotz E, Panknin C. Stress and rest dynamic myocardial perfusion imaging by evaluation of complete time-attenuation curves with dual-source CT. JACC Cardiovasc Imaging 2010;3:811-820.

47. Wang Y, Qin L, Shi X, Zeng Y, Jing H, Schoepf UJ, et al. Adenosine-stress dynamic myocardial perfusion imaging with second-generation dualsource CT: comparison with conventional catheter coronary angiography and SPECT nuclear myocardial perfusion imaging. AJR Am J Roentgenol 2012;198:521-529.

48. Greif M, von Ziegler F, Bamberg F, Tittus J, Schwarz F, D’Anastasi M, et al. CT stress perfusion imaging for detection of haemodynamically relevant coronary stenosis as defined by FFR. Heart 2013;99:1004-1011.

49. Kono AK, Coenen A, Lubbers M, Kurata A, Rossi A, Dharampal A, et al. Relative myocardial blood flow by dynamic computed tomographic perfusion imaging predicts hemodynamic significance of coronary stenosis better than absolute blood flow. Invest Radiol 2014;49:801-807.

50. Rossi A, Dharampal A, Wragg A, Davies LC, van Geuns RJ, Anagnostopoulos C, et al. Diagnostic performance of hyperaemic myocardial blood flow index obtained by dynamic computed tomography: does it predict functionally significant coronary lesions? Eur Heart J Cardiovasc Imaging 2014;15:85-94.

51. Coenen A, Rossi A, Lubbers MM, Kurata A, Kono AK, Chelu RG, et al. Integrating CT myocardial perfusion and CT-FFR in the work-up of coronary artery disease. JACC Cardiovasc Imaging 2017;10:760-770.

52. Coenen A, Lubbers MM, Kurata A, Kono A, Dedic A, Chelu RG, et al. Diagnostic value of transmural perfusion ratio derived from dynamic CTbased myocardial perfusion imaging for the detection of haemodynamically relevant coronary artery stenosis. Eur Radiol 2017;27:2309-2316.

53. Goto Y, Kitagawa K, Uno M, Nakamori S, Ito T, Nagasawa N, et al. Diagnostic accuracy of endocardial-to-epicardial myocardial blood flow ratio for the detection of significant coronary artery disease with dynamic myocardial perfusion dual-source computed tomography. Circ J 2017;81:14771483.

54. Danad I, Raijmakers PG, Appelman YE, Harms HJ, de Haan S, van den Oever ML, et al. Coronary risk factors and myocardial blood flow in patients evaluated for coronary artery disease: a quantitative $[15 \mathrm{O}] \mathrm{H} 2 \mathrm{O}$ PET/CT study. Eur J Nucl Med Mol Imaging 2012;39:102-112.

55. Liga R, Rovai D, Sampietro T, Vecoli C, Todiere G, Caselli C, et al. Insulin resistance is a major determinant of myocardial blood flow impairment in anginal patients. Eur J Nucl Med Mol Imaging 2013;40:1905-1913.
56. Chareonthaitawee P, Kaufmann PA, Rimoldi O, Camici PG. Heterogeneity of resting and hyperemic myocardial blood flow in healthy humans. Cardiovasc Res 2001;50:151-161.

57. Gould KL, Kirkeeide RL, Buchi M. Coronary flow reserve as a physiologic measure of stenosis severity. J Am Coll Cardiol 1990;15:459-474.

58. Kim EY, Chung WJ, Sung YM, Byun SS, Park JH, Kim JH, et al. Normal range and regional heterogeneity of myocardial perfusion in healthy human myocardium: assessment on dynamic perfusion CT using 128-slice dual-source CT. Int J Cardiovasc Imaging 2014;30 Suppl 1:33-40.

59. Ho KT, Ong HY, Tan G, Yong QW. Dynamic CT myocardial perfusion measurements of resting and hyperaemic blood flow in low-risk subjects with 128-slice dual-source CT. Eur Heart J Cardiovasc Imaging 2015;16: 300-306.

60. Fujita M, Kitagawa K, Ito T, Shiraishi Y, Kurobe Y, Nagata M, et al. Dose reduction in dynamic CT stress myocardial perfusion imaging: comparison of $80-\mathrm{kV} / 370-\mathrm{mAs}$ and 100-kV/300-mAs protocols. Eur Radiol 2014;24: 748-755.

61. Kim SM, Kim YN, Choe YH. Adenosine-stress dynamic myocardial perfusion imaging using 128-slice dual-source CT: optimization of the CT protocol to reduce the radiation dose. Int J Cardiovasc Imaging 2013;29: 875-884.

62. Lin L, Wang Y, Yi Y, Cao J, Kong L, Qian H, et al. Application of the lowdose one-stop-shop cardiac CT protocol with third-generation dual-source CT. Zhongguo Yi Xue Ke Xue Yuan Xue Bao 2017;39:34-41.

63. Ho KT, Panknin C. CT myocardial perfusion imaging of myocardial blood flow reserve before and after coronary artery stenting. J Cardiovasc Comput Tomogr 2011;5:125-126.

64. Danad I, Uusitalo V, Kero T, Saraste A, Raijmakers PG, Lammertsma AA, et al. Quantitative assessment of myocardial perfusion in the detection of significant coronary artery disease: cutoff values and diagnostic accuracy of quantitative [(15)O]H2O PET imaging. J Am Coll Cardiol 2014;64: 1464-1475.

65. Kajander SA, Joutsiniemi E, Saraste M, Pietila M, Ukkonen H, Saraste A, et al. Clinical value of absolute quantification of myocardial perfusion with (15)O-water in coronary artery disease. Circ Cardiovasc Imaging 2011;4: 678-684.

66. Gupta A, Taqueti VR, van de Hoef TP, Bajaj NS, Bravo PE, Murthy VL, et al. Integrated noninvasive physiological assessment of coronary circulatory function and impact on cardiovascular mortality in patients with stable coronary artery disease. Circulation 2017;136:2325-2336.

67. Kurobe Y, Kitagawa K, Ito T, Kurita Y, Shiraishi Y, Nakamori S, et al. Myocardial delayed enhancement with dual-source CT: advantages of targeted spatial frequency filtration and image averaging over half-scan reconstruction. J Cardiovasc Comput Tomogr 2014;8:289-298.

68. Aikawa T, Oyama-Manabe N, Naya M, Ohira H, Sugimoto A, Tsujino I, et al. Delayed contrast-enhanced computed tomography in patients with known or suspected cardiac sarcoidosis: a feasibility study. Eur Radiol 2017;27:4054-4063.

69. Tanabe Y, Kido T, Kurata A, Kouchi T, Fukuyama N, Yokoi T, et al. Late iodine enhancement computed tomography with image subtraction for assessment of myocardial infarction. Eur Radiol 2018;28:1285-1292.

70. Kurita Y, Kitagawa K, Kurobe Y, Nakamori S, Nakajima H, Dohi K, et al. Estimation of myocardial extracellular volume fraction with cardiac CT in subjects without clinical coronary artery disease: a feasibility study. J Cardiovasc Comput Tomogr 2016;10:237-241.

71. Lee H-J, Im DJ, Youn J-C, Chang S, Suh YJ, Hong YJ, et al. Myocardial extracellular volume fraction with dual-energy equilibrium contrast-enhanced cardiac CT in nonischemic cardiomyopathy: a prospective comparison with cardiac MR imaging. Radiology 2016;280:49-57. 